\title{
Specific muscle identities are regulated by Krüppel during Drosophila
}

\section{embryogenesis}

\author{
Mar Ruiz-Gómez ${ }^{1}$, Susana Romani' ${ }^{2, \dagger}$, Christine Hartmann ${ }^{3}$, Herbert Jäckle ${ }^{3}$ and Michael Bate ${ }^{1, *}$ \\ ${ }^{1}$ Department of Zoology, University of Cambridge, Downing Street, Cambridge CB2 3EJ, UK \\ 2Department of Anatomy, University of Cambridge, Downing Site, Cambridge CB2 3DY, UK \\ ${ }^{3}$ Abteilung Molekulare Entwicklungsbiologie, Max-Planck-Institut für biophysikalische Chemie, Am Fassberg, D-37077 Göttingen, \\ Germany \\ *Author for correspondence (e-mail: cmb16@cus.cam.ac.uk) \\ †Present address: Centro de Biología Molecular Severo Ochoa, CSIC, Universidad Autónoma de Madrid, Cantoblanco, Madrid 28049, Spain
}

\section{SUMMARY}

During Drosophila embryogenesis, mesodermal cells are recruited to form a complex pattern of larval muscles. The formation of the pattern is initiated by the segregation of a special class of founder myoblasts. Single founders fuse with neighbouring nonfounder myoblasts to form the precursors of individual muscles. Founders and the muscles that they give rise to have specific patterns of gene expression and it has been suggested that it is the expression of these founder cell genes that determines individual muscle attributes such as size, shape, insertion sites and innervation. We find that the segmentation gene Krïppel is expressed in a subset of founders and muscles, regulates specific patterns of gene expression in these cells and is required for the acquisition of proper muscle identity. We show that gain and loss of Krüppel expression in sibling founder cells is sufficient to switch these cells, and the muscles that they give rise to, between alternative cell fates.

Key words: Krüppel, muscle pattern, Drosophila

\section{INTRODUCTION}

During Drosophila embryogenesis, mesodermal cells are recruited to form the elements of a complex pattern of larval muscles. A standard arrangement of 30 muscles develops in each of the abdominal hemisegments A2 to A7, with minor variations in more anterior and posterior segments (Fig. 1). Each muscle is a syncytium that is generated as neighbouring myoblasts fuse to form a multinucleate muscle precursor which then differentiates to form a mature fibre. Every muscle in the pattern is a unique structure, which can be identified by its patterns of gene expression, position, size, shape, insertion sites on the epidermis and innervation. Thus, while all muscles share a set of general muscle properties such as contractile proteins, receptors for neurotransmitters and the capacity to form epidermal attachment sites, each muscle has unique properties that are acquired by the contributing myoblasts and manifested as each muscle precursor differentiates to form a particular muscle fibre (for reviews of Drosophila myogenesis, see Abmayr et al., 1995; Bate, 1993).

There is good evidence that the acquisition of muscle-specific properties during the process of myogenesis depends on the prior specification of a special class of myoblasts called founder cells (Bate, 1990; Dohrmann et al., 1990). During normal embryogenesis, founder cells express genes that are characteristic of muscle subsets in the overall pattern and can be used as markers to chart the development of individual muscle fibres.
Among the genes so far described as being expressed in founders are S59 (Dohrmann et al., 1990), even skipped (eve) (Frasch et al., 1987), vestigial (vg) (Williams et al., 1991), apterous (ap) (Bourgouin et al., 1992), nautilus (nau) (Michelson et al., 1990), connectin (Nose et al., 1992) and Krüppel $(K r)$ (Gaul et al., 1987). Founders fuse with neighbouring myoblasts and recruit them to these patterns of expression. Thus, for example, an S59-expressing founder cell fuses with its neighbours and initiates the formation of a multinucleate S59-expressing muscle precursor (Dohrmann et al., 1990). The unique properties of the founder cells are manifested in mutants where myoblast fusion is blocked: the founders alone express muscle-specific genes such as S59 and develop to form single-celled muscles each of which manifests the special properties of an individual muscle in the normal pattern (Rushton et al., 1995). In the absence of fusion, other myoblasts remain as an undifferentiated set of rounded cells, which may express general muscle characteristics such as the contractile protein myosin. Thus, the founders appear to represent a special class of myoblasts that have access to all the information required to form particular muscles during the process of myogenesis.

Recently, it has been shown that many of the founder cells are derived as pairs of sibling cells from the division of muscle progenitors, which in turn arise from clusters of cells in the mesoderm that express the proneural gene, lethal of scute (Carmena et al., 1995). In the well-analysed case of S59-expressing progenitors and founders, it has been shown that a cluster of 
four distinct founder cells is derived from the division of two progenitors and that, of these, only the precursor derived from one founder maintains $S 59$ expression and goes on to form an S59-expressing muscle (Carmena et al., 1995; Dohrmann et al., 1990). This observation emphasises the likely significance of the patterns of gene expression that have been described for specific muscle subsets and the founder cells that give rise to them. Since sibling founder cells give rise to muscle precursors with distinctly different patterns of gene expression - for example, maintenance of S59 in one or loss from the other - and these in turn give rise to muscles with different characteristics, it seems highly likely that it is the regulated expression of transcription factors such as S59 that conditions the development of some or all of the characteristics of individual muscles.

Although this suggestion has been made many times, it has not so far been possible to demonstrate that altering patterns of gene expression in muscle precursors leads to predictable changes in muscle characteristics. In the case of the apterousexpressing founders and precursors, it was possible to show that loss of function could eliminate some of the apterousexpressing muscles and that ectopic apterous expression could lead to a duplication of some of the normally apterous-expressing muscles (Bourgouin et al., 1992). Similar results were obtained after overexpression of nautilus in the mesoderm (Keller et al., 1997). While these results are interesting, they do not specifically address the important question of whether altering patterns of gene expression in muscle precursors can lead to predictable changes in the differentiation of muscles. In particular, it is a prediction of this view of myogenesis that it should be possible to transform individual muscle phenotypes by switching patterns of gene expression from those characteristic of one precursor to those typical of another.

In this paper, we describe the role of the gene Krüppel $(\mathrm{Kr})$ in the development of a subset of the somatic muscles. Although the expression of $K r$ in the mesoderm and in the somatic muscles has been described previously (Gaul et al., 1987), it has not been possible to look directly at the requirement for $K r$ in myogenesis, because of the earlier requirement for $K r$ in establishing the embryonic body plan. Here we use a $K r$ construct that rescues the early $K r$ phenotype by promoting $K r$ expression in the central body region of the early embryo (Romani et al., 1996). At later stages, however, there is no $K r$ expression in the CNS or the mesoderm and we take advantage of this fact to look at the effects of loss of $K r$ function on the differentiation of $K r$-expressing muscles. Our results indicate that $K r$ is required for the maintenance of expression of genes such as S59 in those precursors where $K r$ is expressed and that these patterns of gene expression are necessary for the acquisition of specific muscle characteristics. Loss of $\mathrm{Kr}$ leads to a premature loss of expression of muscle-specific genes and this in turn is accompanied by clear muscle transformation. Ectopic $K r$ expression does not lead to the activation of genes such as $S 59$, but maintains the expression of $S 59$ and other genes in precursors from which they are normally lost, again leading to muscle transformation.

\section{MATERIALS AND METHODS}

\section{Drosophila strains}

The following strains of flies were used in this work: Oregon $R, \mathrm{Kr}^{\mathrm{CD}+}$ $\mathrm{Kr}^{\mathrm{l}} / \mathrm{CyO} \mathrm{Ob}$-lacZ; ry (a transgenic line that rescues the $\mathrm{Kr}$ segmentation phenotype, described in Romani et al., 1996), UASKr ;UASKr (a generous gift from Michael Hoch and Alan Michelson) and twiGALA; 24B-GAL4 (described in Baylies and Bate, 1996).

\section{Histochemistry}

In situ hybridizations in whole-mount embryos were performed with minor modifications to the protocol of Tautz and Pfeifle (1989). Immunological stainings of whole-mount embryos, using the Vectastain ABC Elite Kit from Vectalabs were made as described in RuizGómez and Ghysen (1993). Stained embryos were embedded in Araldite and sectioned $(5 \mu \mathrm{m})$ following standard procedures. The following primary antibodies were used: anti-Krüppel (Gaul et al., 1987), anti- $\beta$-galactosidase (Cappel), anti-muscle Myosin (Kiehart and Feghali, 1986), anti-S59 (Dohrmann et al., 1990), anti-Connectin (Nose et al., 1992), anti-Eve (Patel et al., 1994), anti-Fasciclin II (Van Vactor et al., 1993) and anti-Vestigial (Williams et al., 1991). Antibodies were used at the same concentration described in Rushton et al. (1995). Stained embryos were examined and photographed using a Zeiss Axiophot microscope.

Immunofluorescent stainings were performed as in Carmena et al. (1995). Fluorescent images were recorded by use of a Zeiss LSM 310 confocal microscope.

\section{Ectopic expression}

Ectopic expression of $K r$ was generated by means of the GAL4 targeted expression system (Brand and Perrimon, 1993) In those experiments, males bearing four copies of $U A S K r$ were crossed to twiGAL4; $24 B G A L 4$ females at $29^{\circ} \mathrm{C}$. Under these conditions, generalised ectopic expression of $K r$ is induced in the mesoderm from stage 9 until the end of embryogenesis (not shown).

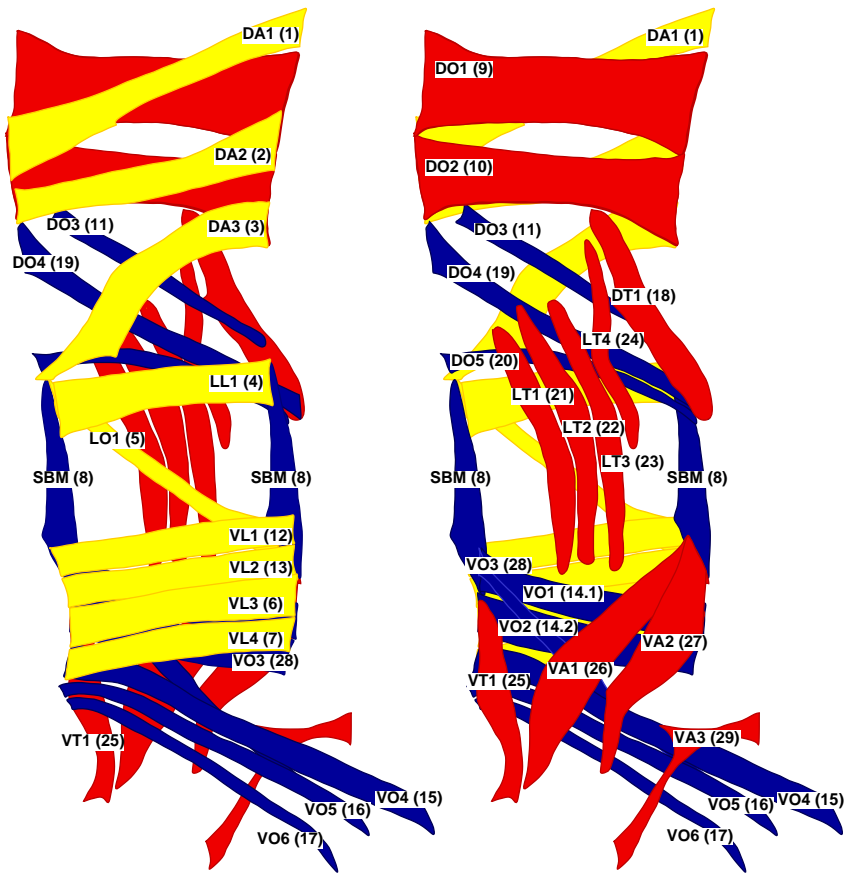

Fig. 1. Diagram showing internal (left) and external (right) views of the larval muscle pattern of abdominal segments A2-A7. External muscles are shown in red and more internal ones in blue and yellow. Dorsal is up and anterior is to the left. Each muscle is identified according to the scheme described in Bate (1993) and by the number given by Crossley (1978). DA, dorsal acute; DO, dorsal oblique; DT, dorsal transverse; LO, lateral oblique; LT, lateral transverse; LL, lateral longitudinal; VA, ventral acute; VO, ventral oblique; VT, ventral transverse; VL, ventral longitudinal; SBM, segment border muscle. 


\section{RESULTS}

\section{Kr expression during muscle development}

The segmentation gene Krüppel $(K r)$ encodes a transcriptional regulator that, besides its early function during segmentation, is also expressed in the precursors of a subset of body wall muscles (Gaul et al., 1987). To investigate the role of $K r$ in myogenesis, we made a more precise description of its normal pattern of expression in muscle-forming cells by in situ hybridizations with a $K r$ cDNA probe and by stainings with $\mathrm{Kr}$ antibodies. Initially, $\mathrm{Kr}$ is detected in clusters of cells in the mesoderm (Fig. 2A), and then in a subset of the muscle progenitors (Fig. 2B). Each of these progenitors divides to give rise to two founder cells, both of which express $K r$ (Fig. 2C). In normal embryos, the expression of $K r$ is always lost from one of the two founders before it fuses with neighbouring myoblasts to form a syncytial muscle precursor. The other founder maintains $K r$ expression and fuses with surrounding myoblasts to form a $K r$-expressing muscle precursor (Fig. 2D). Thus, an early distinction between the two muscle founders arising from a single $\mathrm{Kr}$-expressing progenitor is the maintenance of $K r$ expression in only one of them. We have relied on the late pattern of expression to identify individual $K r$-expressing muscles by their position and/or by double staining with antibodies to other markers such as Connectin (Nose et al., 1992). $K r$ protein is expressed in two dorsal muscles (for nomenclature see Bate, 1993 and Fig. 1): the dorsal acute muscle 1 (DA1) and the dorsal oblique muscle 1 (DO1), in three lateral muscles including the lateral longitudinal muscle 1 (LL1) and the lateral transverse muscles 2 and 4 (LT2, LT4) and in four ventral muscles including ventral longitudinal muscle 3 (VL3), ventral acute muscle 2 (VA2) and the ventral oblique muscles 2 and 5 (VO2, VO5) (Fig. 2D). During early stage 14 $K r$ is also expressed transiently in the ventral longitudinal muscles 2 and 4 (VL2, VL4). Both $K r$ transcripts and protein decrease during stage 15 and are absent from differentiated muscle fibres.

\section{Requirement for $K r$ during myogenesis}

$K r$ lack-of-function alleles cause strong segmentation defects (Wieschaus et al., 1984). Thus, any muscle defects observed in $K r$ embryos are likely to be a consequence of the abnormal segmentation process. To circumvent this difficulty and assess the role of $K r$ in the mesoderm, we examined the muscle pattern in embryos that carried a $K r$ transgene providing early $K r$ expression. Provision of the transgene specifically rescues the segmentation defect of $K r$ lack-of-function mutants (Romani et al., 1996). We refer to embryos containing two copies of this $K r$ minigene as ' $K r^{\mathrm{CD}}{ }^{\mathrm{C}} \mathrm{Kr}{ }^{1}$ embryos'.

The $K r^{\mathrm{CD}+} K r^{1}$ embryos show severe and consistent disruptions to the muscle pattern, which are confined to the $K r$ expressing muscle subset. We grouped the $K r$-positive muscles into three different categories according to the kind of defects found in $K r^{\mathrm{CD}+} \mathrm{Kr}^{1}$ embryos (Table 1). The first class (muscles DO1 and VL3) is apparently unaffected. The second class (muscles DA1, LL1, VO2 and VA2) includes muscles that are present but whose morphology and/or orientation are frequently abnormal. Muscles of the third class (LT2, LT4 and VO5) are occasionally absent from the final pattern and can be transformed (see Table 1; Fig. 6E,F). The fact that there is a consistent pattern of muscle defects in the $K r^{\mathrm{CD}+} \mathrm{Kr}^{1}$ embryos,

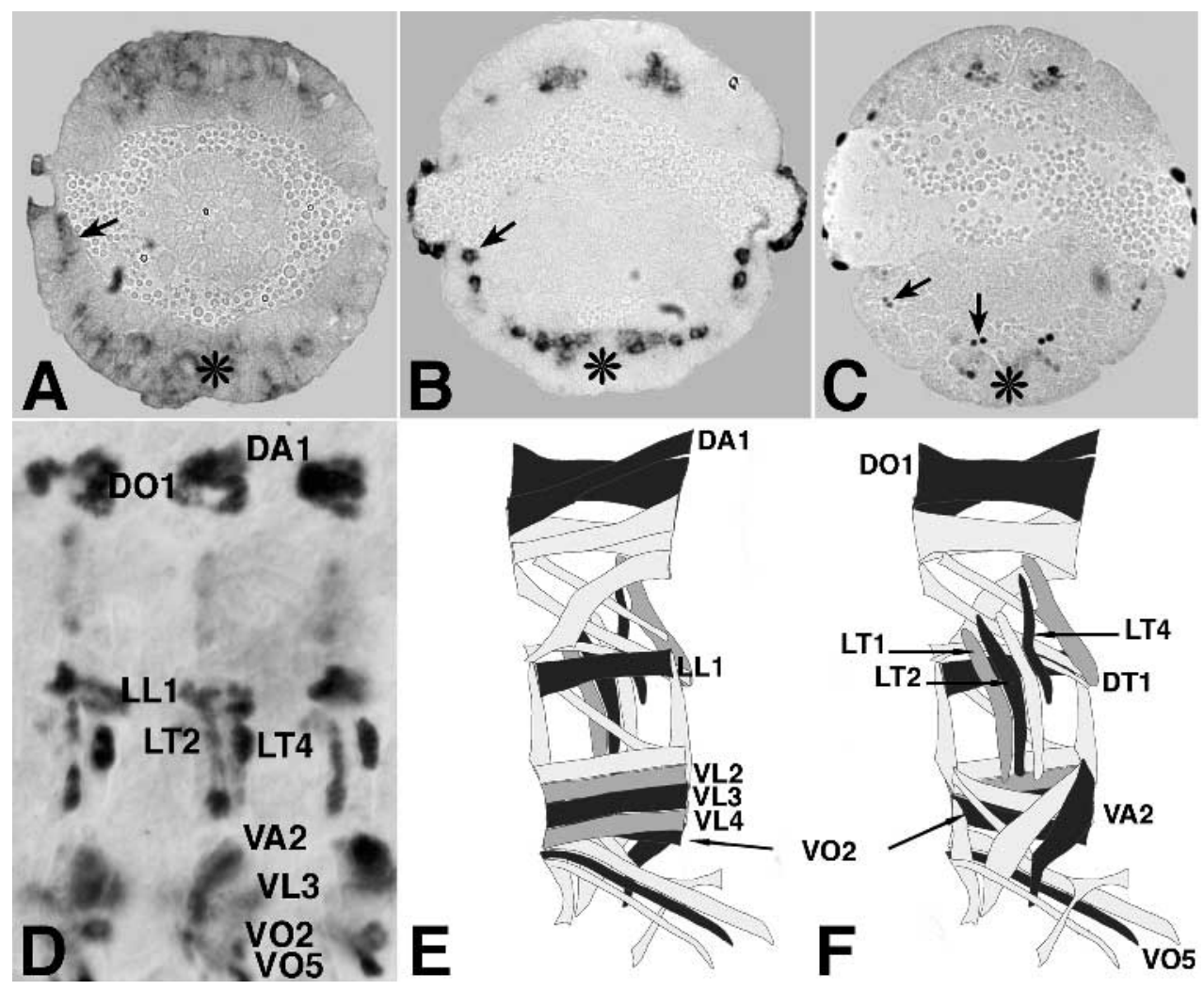

Fig. 2. Expression of $K r$ in the mesoderm. (A-C) Sectioned embryos after in situ hybridization with $K r$ probes (A,B) and after anti-Kr antibody staining (C). (A) Early stage 11 embryo showing $\mathrm{Kr}$ expression in a cluster of cells located on the dorsal edge of the mesoderm (arrow).

(B) Accumulation of $K r$ expression in the progenitors of somatic muscles in late stage 11 embryo. Arrow points to a progenitor that has segregated from the cell cluster shown in A. (C) By stage 12, $\mathrm{Kr}$ is detected in the two founder cells resulting from the division of the progenitors (arrows). (A-C) The asterisk is on the CNS. (D) Stage 14 embryo stained with anti-Kr antibodies and opened flat to show $K r$-expressing muscle precursors. Drawings of internal (E) and external (F) view of the muscle pattern of abdominal segments A2-7. The $K r$-expressing muscles are shown in black (dark grey indicates transient or low expression). (D-F) Anterior is to the left and dorsal is up. DA1, dorsal acute 1; DO1, dorsal oblique 1; DT1, dorsal transverse 1; LL1, lateral longitudinal 1; LT1, LT2, LT4, lateral transverse 1, 2, 4; VL2, VL3, VL4, ventral longitudinal 2, 3, 4; VA2, ventral acute 2; VO2, VO5, ventral oblique 2,5 . 
Fig. 3. Evolution of the patterns of expression of $K r$ and S59 in the developing muscles VA1 and VA2. (A-D) Confocal micrographs of embryos double stained with anti-Krüppel (green) and anti-S59 (red) antibodies. (A) Ventral view of a late stage 11 embryo showing coexpression of $K r$ and $S 59$ in the progenitor of muscles VA1 and VA2 (arrowhead). $S 59$ but not $K r$ is expressed in the progenitor of muscle VA3 and ventral adult precursor (arrow). The bar indicates the ventral midline. (B) Lateral view of a stage 12 embryo showing that both transcription factors are coexpressed in the VA1 and VA2 founders (arrowheads). (C) $K r$ expression declines from the VA1 founder by late stage 12 before it fuses to neighbouring myoblasts (arrowhead). (D) By stage 14 the VA2 precursor is the only one that coexpresses $K r$ and S59 (arrowhead). (B-D) Ventral is to the bottom and anterior is to the left.
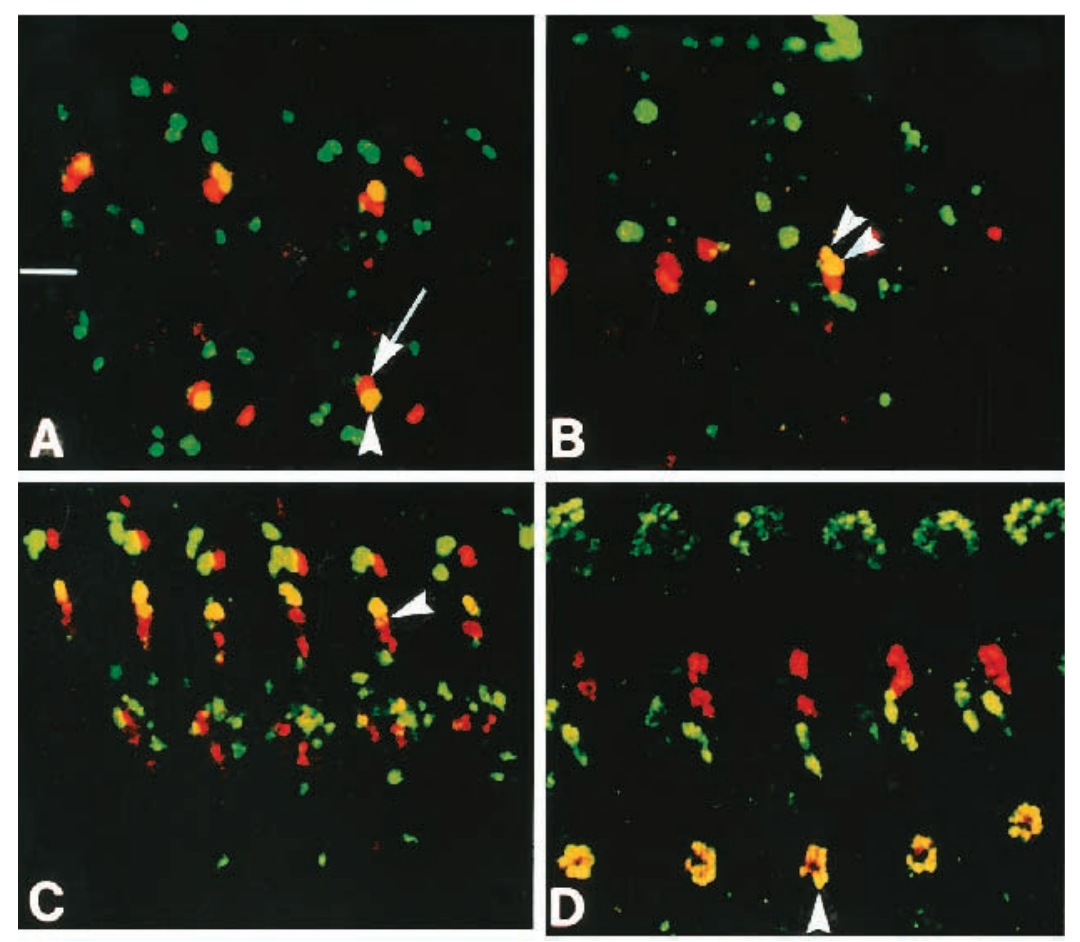

which exclusively affects the normally $K r$-expressing muscles, strongly suggests that $K r$ function may be required for the normal development of this subset of the muscles.

\section{Development of VA1 and VA2 muscles in the absence of Krüppel and in the presence of ectopic Krüppel}

A characteristic feature of Krüppel expression is its differential regulation between the two founder cells arising from $\mathrm{Kr}$ expressing progenitors. In those cases that we have studied, $\mathrm{Kr}$ expression is maintained in only one of the founders and the precursors that they give rise to and the muscles derived from these precursors are precisely those that are modified in $K r^{\mathrm{CD}+} \mathrm{Kr}^{1}$ embryos.

We reasoned that the differential expression of $K r$ in the two founders might play a role in conferring distinctive characteristics on the resulting muscles and their precursors. To test this idea, we focused on the single muscle progenitor that coexpresses $\mathrm{Kr}$ and the homeodomain protein S59 (Fig. 3A) and the two sibling founder cells that it gives rise to. These cells are the founders of adjacent, but different muscles VA1 and VA2 (Fig. 4). The VA1 muscle is formed close to VA2, but the two fibres have different orientations and VA1 inserts more anteriorly in the epidermis.

Initially both the VA1 and VA2 founders coexpress $\mathrm{Kr}$ and S59 (Fig. 3B) but, as development proceeds, the expression of both genes disappears from VA1. $\mathrm{Kr}$ expression is lost first, soon after the VA1 founder is formed (Fig. 3C), and S59 disappears once VA1 has fused with neighbouring cells to form a recognisable precursor (Fig. 3D). By contrast, the sibling founder cell (VA2) maintains $S 59$ and $K r$ expression and gives rise to a muscle precursor that expresses both genes (Fig. 3D). $S 59$ continues to be expressed in the differentiated VA2 muscle, but $\mathrm{Kr}$ expression is lost. Thus, a unique progenitor in which $\mathrm{Kr}$ and $\mathrm{S59}$ are coexpressed gives rise to two muscles with distinct patterns of gene expression and final morphology (Figs $4,5)$.

To show whether $K r$ has a function in determining the different fates of VA1 and VA2, we first looked at the effects of loss of $K r$, using $K r^{\mathrm{CD}+} K r^{1}$ embryos. In such embryos, the expression of $S 59$ is initiated normally in the VA1/2 progenitor (Figs 4A,B, 5), but is no longer maintained in the VA2 precursor (Figs 4D,G, 5). When we examine the muscle that now forms at the VA2 position, it is transformed and has the characteristics of VA1 so that two muscles with the orientations and insertion sites of VA1 are now present (Figs 4J, 5). These findings suggest that $K r$ is required to maintain $S 59$ expression in the VA2 precursor and that loss of $K r$ from VA2 alters its developmental fate into that of a muscle that expresses neither $\mathrm{Kr}$ nor $\mathrm{S59}$. If this is so, then the distinc-

Table 1. Phenotypes produced by the loss of Krüppel in the mesoderm in $\mathrm{Kr}^{\mathrm{CD}+} \mathrm{Kr}^{I}$ mutant embryos

\begin{tabular}{lcccc}
\hline Class & Muscle & \% Presence* & $\begin{array}{c}\text { \% Morphological } \\
\text { changes } \dagger\end{array}$ & $\begin{array}{c}\text { \% Changes in } \\
\text { gene expression }\end{array}$ \\
\hline I & DO1 & 100 & 3 & - \\
II & VL3 & 100 & 0 & - \\
& DA1 & 90 & 80 & $100($ eve, vg) \\
& LL1 & 90 & 50 & - \\
& VA2 & 98 & 70 & $100(\mathrm{~s} 59)$ \\
III & VO2 & 100 & 100 & - \\
& LT1-4 & $70 \ddagger$ & $50 \S$ & -
\end{tabular}

*'Presence' indicates that a muscle forms in experimental embryos at the position normally occupied by the muscles concerned in wild-type embryos. $\dagger$ Refers to changes in shape, orientation or attachment sites.

†LT1-4 and VO4-6 can not be identified individually.

\$Whenever muscle LT4 is present its attachment sites are changed to those of muscles LT1-3.

- , not determined. 
tion between the alternative fates of the two sibling cells (VA1 and VA2) depends on the restriction of $K r$ expression to one of them. We tested this by analysing the effects of maintained $K r$ expression on the fate of the VA1 and VA2 founders. When the Gal4 system is used to express $K r$ throughout the mesoderm (Brand and Perrimon, 1993) and therefore in both VA1 and VA2, S59 expression is initiated normally but is then maintained in the VA1 precursor from which it would normally be lost (Figs 4F,I,L, 5). Furthermore, the opposite transformation now occurs and two muscles with the orientation and insertion sites of VA2 are now formed (Figs 4L, 5).

\section{Loss of $K r$ leads to failures in the maintenance of specific patterns of gene expression and to muscle transformations}

It may be that other muscle transformations that occur consistently in the $K r^{\mathrm{CD}+} K r^{1}$ embryos are similarly caused by a failure of $K r$ to maintain normal patterns of gene expression. For example, in $K r^{\mathrm{CD}}{ }^{\mathrm{N}} r^{1}$ embryos, DA 1 is routinely transformed to either an oblique or a transverse muscle at the DA1 position (Fig. $6 \mathrm{~B})$. This transformation is associated with the loss of eve and $v g$ expression from the DA1 precursor (Fig. 6D and data not shown). We also observe the formation of an ectopic VL muscle in $\mathrm{Kr}^{\mathrm{CD}+} \mathrm{Kr}^{1}$ embryos (Fig. 6G). This additional VL muscle expresses $v g$, like its neighbours, and is always associated with the loss of the adjacent VO2 muscle. Occasionally, there appears to be a partial transformation of $\mathrm{VO} 2$ towards the VL fate - in these cases, $\mathrm{VO} 2$ is missing and an intermediate muscle with both oblique and longitudinal insertions is seen in its place (data not shown). Thus, in this instance, it seems likely that loss of $K r$ from

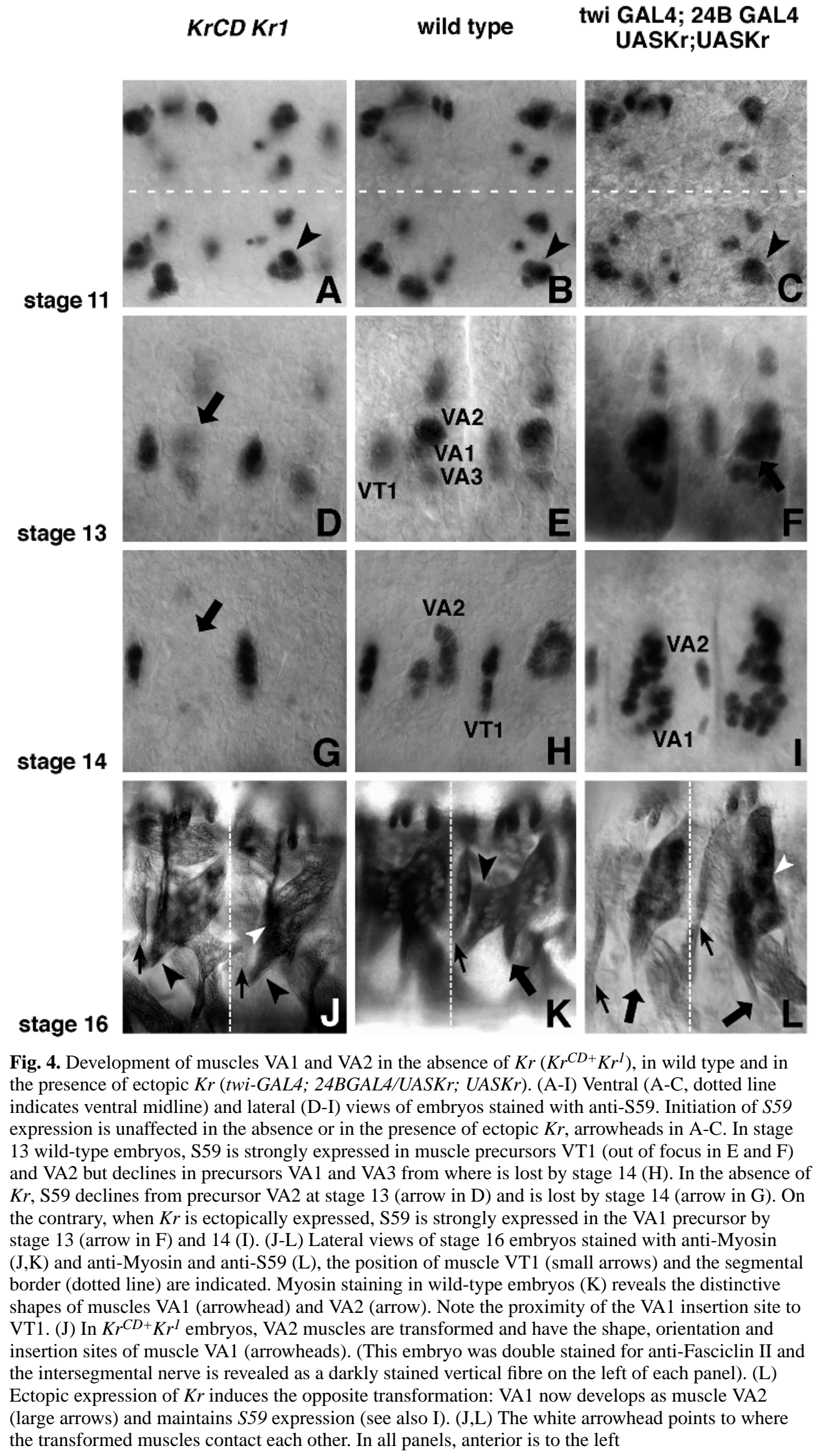

KrCD Kr1

stage 14

Fig. 4. Development of muscles VA1 and VA2 in the absence of $K r\left(K r^{C D+} K r^{l}\right)$, in wild type and in the presence of ectopic $K r$ (twi-GAL4; 24BGAL4/UASKr; UASKr). (A-I) Ventral (A-C, dotted line indicates ventral midline) and lateral (D-I) views of embryos stained with anti-S59. Initiation of S59 expression is unaffected in the absence or in the presence of ectopic $K r$, arrowheads in A-C. In stage 13 wild-type embryos, S59 is strongly expressed in muscle precursors VT1 (out of focus in E and F) and VA2 but declines in precursors VA1 and VA3 from where is lost by stage $14(\mathrm{H})$. In the absence of $K r$, S59 declines from precursor VA2 at stage 13 (arrow in D) and is lost by stage 14 (arrow in G). On the contrary, when $K r$ is ectopically expressed, S59 is strongly expressed in the VA1 precursor by stage 13 (arrow in F) and 14 (I). (J-L) Lateral views of stage 16 embryos stained with anti-Myosin $(\mathrm{J}, \mathrm{K})$ and anti-Myosin and anti-S59 (L), the position of muscle VT1 (small arrows) and the segmental border (dotted line) are indicated. Myosin staining in wild-type embryos (K) reveals the distinctive shapes of muscles VA1 (arrowhead) and VA2 (arrow). Note the proximity of the VA1 insertion site to VT1. (J) In $\mathrm{Kr}^{\mathrm{CD}}{ }^{\mathrm{K}} \mathrm{r}^{1}$ embryos, VA2 muscles are transformed and have the shape, orientation and insertion sites of muscle VA1 (arrowheads). (This embryo was double stained for anti-Fasciclin II and the intersegmental nerve is revealed as a darkly stained vertical fibre on the left of each panel). (L) (large arrows) and maintains 559 expression (see also I). (J,L) The white arrowhead points to where the transformed muscles contact each other. In all panels, anterior is to the left 
the normally $\mathrm{Kr}$-expressing founder and precursor of $\mathrm{VO} 2$ leads to the transformation of this muscle to a VL fate.

In summary, using a number of different markers, we find that $K r$ is not required for the segregation of a normal pattern of muscle founder cells or to initiate patterns of gene expression in these founders, but it is required for the maintenance of normal patterns of gene expression in the precursors that these founders form. Therefore the effects of $K r$ on muscle pattern are not to do with the segregation of founders but may be connected with the failure to maintain normal patterns of expression in genes such as $S 59$.

Fig. 5. Diagram showing the consequences of lack and excess of function of $K r$ in the development of muscles VA1-3 and the adult ventral muscle precursor (AP). Light and dark shading indicates levels of S59, $K r$ expression is represented by a black outline. Only the cells that can be identified by S59 or Myosin (muscles, bottom line) expression are represented. During normal development, two S59-positive progenitors give rise to the three S59-positive founders that will seed the formation of muscles VA1-3 and to the AP. Only the more dorsal progenitor and its two founders will express $\mathrm{Kr}$. Kr is lost in VA1 founder and S59 decays in precursors VA1 and VA3 and in AP, whereas both $\mathrm{S} 59$ and $\mathrm{Kr}$ are uniquely maintained in the VA2 precursor. In the absence of $K r$, the segregation of S59-positive progenitors and founders is not affected. However, S59 expression declines in all the precursors by stage 13 , indicating that the maintenance and not the initiation of S59 expression in VA2 is dependent on $\mathrm{Kr}$. In these conditions, VA2 is transformed towards its non-S59 expressing sibling VA1. When $\mathrm{Kr}$ is ectopically provided in the whole mesoderm, the segregation of $S 59$-expressing cells is unaffected, confirming that $\mathrm{Kr}$ is unable to initiate $S 59$ expression. Furthermore, there is no effect on S59 expression in those cells where it is not normally expressed (VA3 and AP). However, S59 is maintained in VA1 precursor and muscle, which is transformed towards the S59-expressing VA2 fate.

Fig. 6. Phenotypes produced by the absence of $\mathrm{Kr}$ in the mesoderm. Stage 16 wild-type (A) and $K r^{C D+} K r^{l}$ (B) embryos stained with anti-Myosin to reveal the transformation of muscle DA1 into transverse (arrow) or oblique muscle (arrowheads). The dorsal vessel in A and B is out of focus (asterisks). (C,D) Stage 14 wild-type and $K r^{C D+} K r^{1}$ embryos stained with anti-Eve to show that the transformed DA1 muscles do not express Eve (D). Arrow in C points to an eve-expressing DA1 muscle. The pericardial cells continue to express Eve in $\mathrm{Kr}^{\mathrm{CD}}{ }^{\mathrm{K}} \mathrm{Kr}^{1}$ embryos (arrowhead in D). Stage 16 wild-type (E) and $K r^{C D+} K r^{l}(\mathrm{~F})$ embryos stained with anti-Myosin (or anti-Myosin and anti-FasII in F). In $\mathrm{Kr}^{\mathrm{CD}+} \mathrm{Kr}^{1}$ embryos, the LT group frequently contains less muscles (asterisk in F). When 4 muscles are present, the attachment sites of LT4 are changed to those of muscles LT1-3 (arrows in E and F). (G) Stage $16 \mathrm{Kr}^{\mathrm{CD}+} \mathrm{Kr}^{1}$ embryo dissected flat to show the ectopic VL muscle. In most segments, the ectopic muscle develops in an intermediate position and it is masked by the VL2 muscle. In the segment indicated by an asterisk, the ectopic VL muscle runs in parallel to the VL 1-4 muscles. In all panels, anterior is to the left and dorsal is up.

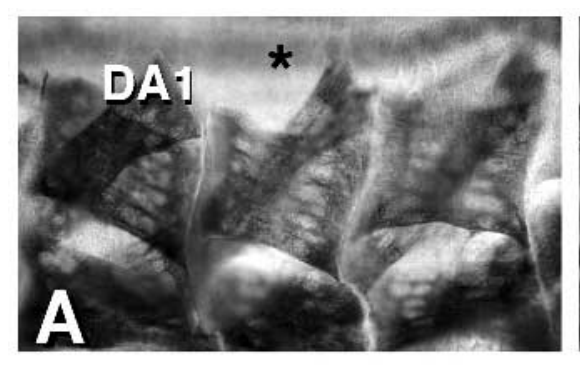

wt

twi Gal4;24B GAL4

UASKr; UASKr
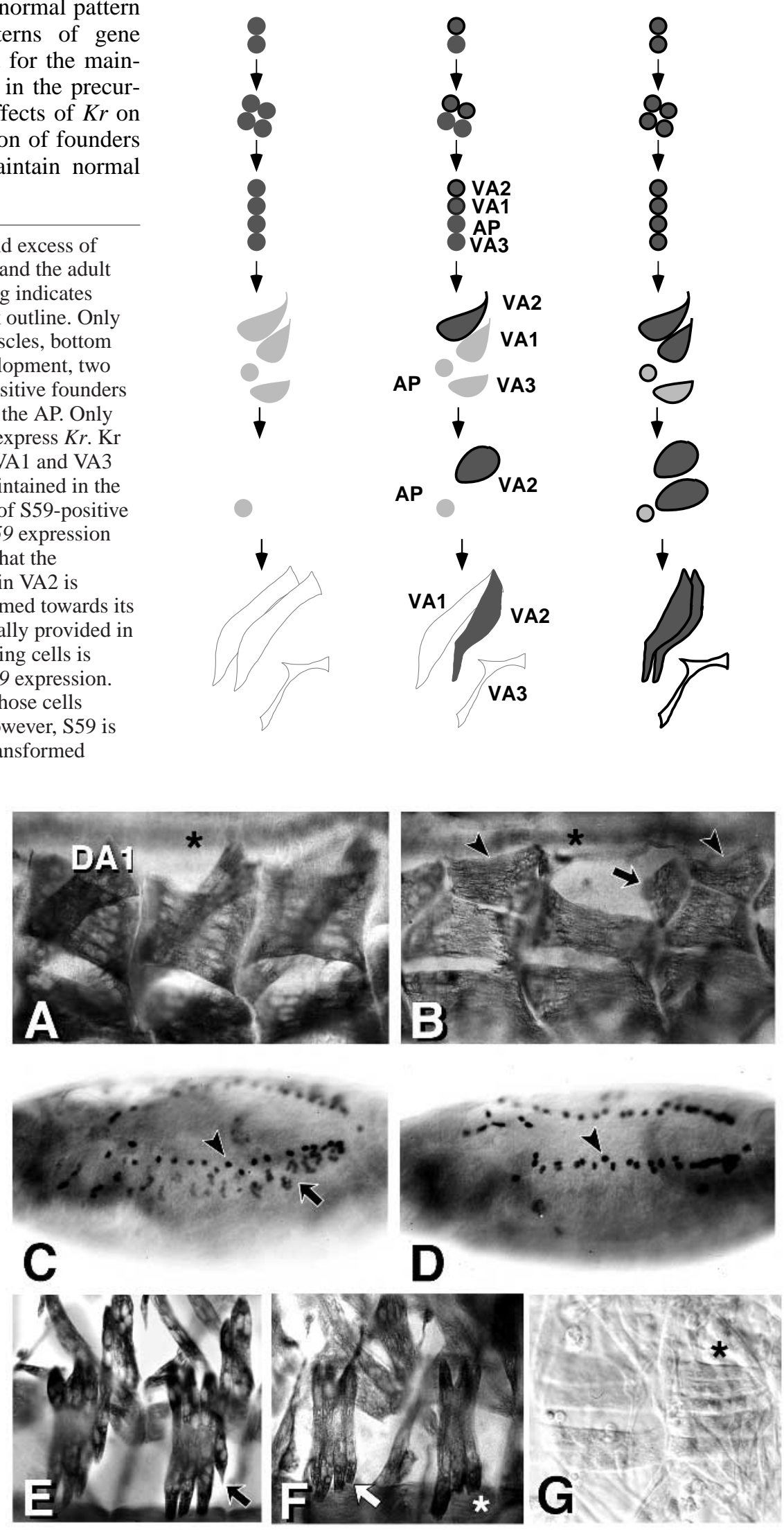
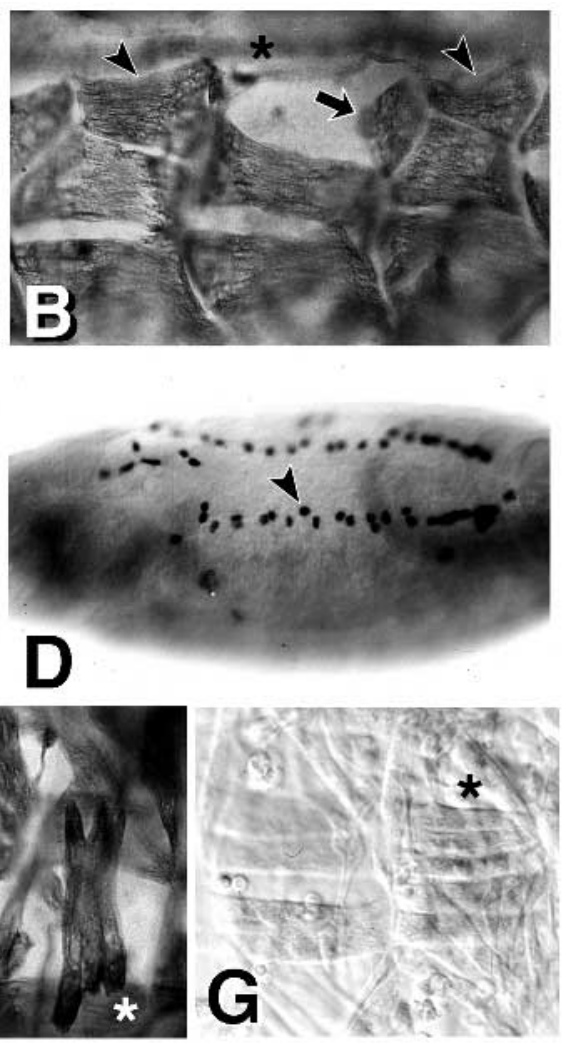


\section{DISCUSSION}

Because a great deal of our understanding of myogenesis and its control comes from experiments on cultured cells, rather little is known about the mechanisms that underlie the spatial organisation of diverse muscles into patterns. However, the acquisition of specific muscle properties (such as size, shape, orientation) implies that the general pathway of myogenesis leading to the synthesis of skeletal muscle proteins is conditioned by extrinsic or intrinsic factors that regulate the differentiation of individual fibres. In Drosophila, there is circumstantial evidence to suggest that the expression of genes such as $K r$ and $S 59$ in subsets of the developing muscles and their precursors may regulate the differentiation of specific muscles (Abmayr et al., 1995). In vertebrates too, the homeobox gene $N k x-2.5$ is expressed in the myocardial lineage (Lints et al., 1993) and engrailed is expressed in muscle pioneers of the axial musculature as well as the progenitors of specific jaw muscles in zebrafish (Hatta et al., 1990). These findings, together with evidence for enhancer activity that is muscle specific (Patapoutian et al., 1993; Shield et al., 1996) suggest that a similar regulatory pathway can operate in vertebrates. Our experiments have tested this hypothesis in Drosophila by altering the expression of $\mathrm{Kr}$ in the mesoderm and showing that the presence or absence of the $\mathrm{Kr}$ protein is sufficient to switch muscles derived from sibling founder cells between alternative fates.

These experiments not only show that local expression of factors such as $\mathrm{Kr}$ in the myogenic lineage can regulate the diversification of muscles but also provide some insight into the way in which such factors may interact with the myogenic pathway in general. One possibility is that the expression of genes such as $\mathrm{Kr}$ and $S 59$ is necessary for the formation of individual muscles. However our experiments show that loss of $\mathrm{Kr}$ does not prevent muscle differentiation, but alters the specific characteristics of individual muscles in which it is normally expressed. Thus $K r$ acts in concert with the myogenic pathway to define specific muscle properties; it does not control myogenesis itself. Our evidence suggests that $K r$ exerts its effect by regulating the expression of genes such as $S 59$ in specific muscle precursors. The fact that ubiquitous expression of $\mathrm{Kr}$ in the mesoderm does not alter the pattern of $\$ 59$ expression in muscle progenitors (Fig. 4C) and that the onset of the 559 expression is normal in the absence of $K r$ shows that, while $K r$ is required for the maintenance of $S 59$ in specific muscleforming cells, it is not involved in its initiation. The same is true for $\mathrm{Kr}$-dependent gene expression in other muscles (eve and $v g$ in DA1, see above).

In the case of the VA1/2 progenitor and founders, it is unlikely that differences in the expression of $S 59$ alone are sufficient to account for the alternative fates adopted by the two cells. $K r$ is known to regulate the expression of a second gene knockout (ko) (Hartmann et al., 1997) that is differentially expressed in VA1 and VA2 and is required for the development of a normal pattern of motor innervation. It is likely that $\mathrm{Kr}$ regulates a number of different genes, such as $S 59$ and $\mathrm{ko}$, and that it is the coordinated expression of these genes, which, in turn, controls specific characteristics such as insertion sites and innervation. However, these data provide the first demonstration that genes expressed in subsets of founder cells can regulate muscle identity and that the differential expression of such genes in the founders produced by a single muscle progenitor switches these cells into different developmental fates.

We thank M. Landgraf for kindly providing figure 1 and D. P. Kiehart for the anti-muscle Myosin antibody. We also thank A. Carmena for help with confocal microscopy and M. Baylies, J. de Celis, A. Martinez-Arias and H. Skaer for comments on the manuscript. This work was supported by grants from the Wellcome Trust (M. B.) and the BBSRC (S. R., who is grateful for being able to carry out this work in the laboratory of S. J. Bray) and by the Max Planck Society (C. H. and H. J.).

\section{REFERENCES}

Abmayr, S. M., Erickson, M. S. and Bour, B. A. (1995). Embryonic development of the larval body wall musculature of Drosophila melanogaster. Trends Genet. 11, 153-159.

Bate, M. (1990). The embryonic development of larval muscles in Drosophila. Development 110, 791-804.

Bate, M. (1993). The mesoderm and its derivatives. In The Development of Drosophila melanogaster, vol. 2 (ed. M. Bate and A. Martinez-Arias), pp 1013-1090. Cold Spring Harbor, New York: CSH Laboratory Press.

Baylies, M. K. and Bate, M. (1996). twist: A myogenic Switch in Drosophila. Science 272, 1481-1484.

Bourgouin, C., Lundgren, S. E. and Thomas, J. B. (1992). apterous is a Drosophila LIM domain gene required for the development of a subset of embryonic muscles. Neuron 9, 549-561.

Brand, A. H. and Perrimon, N. (1993). Targeted gene expression as a means of altering cell fates and generating dominant phenotypes. Development 118, 401-415.

Carmena, A., Bate, M. and Jiménez, F. (1995). lethal of scute, a proneural gene, participates in the specification of muscle progenitors during Drosophila embryogenesis. Genes Dev. 9, 2373-2383.

Crossley, A. C. (1978). The morphology and development of the Drosophila muscular system. In The Genetics and Biology of Drosophila, vol. 2b (ed. M. Ashburner and T. Wright), pp. 499-560. New York: Academic Press.

Dohrmann, C., Azpiazu, N. and Frasch, M. (1990). A new Drosophila homeobox gene is expressed in mesodermal precursor cells of distinct muscles during embryogenesis. Genes Dev. 4, 2098-2111.

Frasch, M., Hoey, T., Rushlow, C., Doyle, H. J. and Levine, M. (1987) Characterization and localization of the even-skipped protein of Drosophila. EMBO J. 6, 749-759.

Gaul, U., Seifert, E., Schuh, R. and Jäckle, H. (1987). Analysis of Krüppel protein distribution during early Drosophila development reveals posttranscriptional regulation. Cell 50, 639-647.

Hartmann, C., Landgraf, M., Bate, M. and Jäckle, H. (1997). Krüppeldependent knockout activity is required for proper innervation of a specific set of Drosophila larval muscles. EMBO J. (in press).

Hatta, K., Schilling, T. F., BreMiller, R. A. and Kimmel, C. B. (1990) Specification of jaw muscle identity in zebrefish: correlation with engrailedhomeodomain expression. Science 250, 802-805.

Keller, C. A., Erickson, M. S. and Abmayr, S. M. (1997). Misexpression of nautilus induces myogenesis in cardioblasts and alters the pattern of somatic muscles fibers. Dev. Biol. 181, 197-212.

Kiehart, D. P. and Feghali, R. (1986). Cytoplasmic myosin from Drosophila melanogaster. J. Cell Biol. 103, 1517-1525.

Lints, T. J., Parsons, L. M., Hartley, L., Lyons, I. and Harvey, R. P. (1993). $N k x$-2.5: a novel murine homeobox gene expressed in early heart progenitor cells and their myogenic descendants. Development 119, 419-431.

Michelson, A. S., Abmayr, S., Bate, M., Martinez Arias, A. and Maniatis, T. (1990). Expression of a Myo-D family member prefigures muscle pattern in Drosophila embryos. Genes Dev. 4, 2086-2097.

Nose, A., Mahajan, V. B. and Goodman, C. S. (1992). Connectin: A homophilic cell adhesion molecule expressed on a subset of muscles and the motoneurons that innervate them in Drosophila. Cell 70, 553-567.

Patapoutian, A., Miner, J. H., Lyons, G. E. and Wold, B. (1993). Isolated sequences from the linked myf-5 and MRF4 genes drive distinct patterns of muscle specific expression in transgenic mice. Development 118, 61-69.

Patel, N. H., Condron, B. G. and Zinn, K. (1994). Pair rule expression patterns of even-skipped are found in both short- and long-germ beetles. Nature 367, 429-432. 


\section{M. Ruiz-Gómez and others}

Romani, S., Jimenez, F., Hoch, M., Patel, N. H., Taubert, H. and Jäckle, H. (1996). Krüppel, a Drosophila segmentation gene, participates in the specification of neurons and glial cells. Mech. Dev. 60, 95-107.

Ruiz-Gómez, M. and Ghysen, A. (1993). The expression and role of a proneural gene, achaete, in the development of the larval Drosophila nervous system. EMBO J. 12, 1221-1230.

Rushton, E., Drysdale, R., Abmayr, S. M., Michelson, A. M. and Bate, M. (1995). Mutations in a novel gene, myoblast city, provide evidence in support of the founder cell hypothesis for Drosophila muscle development. Development 121, 1979-1988.

Shield, M. A., Haugen, H. S., Clegg, C. H. and Hauschka, S. D. (1996). Ebox sites and a proximal regulatory region of the muscle creatine kinase gene differentially regulate expression in diverse skeletal muscles and cardiac muscle of transgenic mice. Mol. Cell. Biol. 16, 5058-5068.

Tautz, D. and Pfeifle, C. (1989). A non-radioactive in situ hybridization method for localization of specific RNAs in Drosophila embryos reveals transcriptional control of the segmentation gene hunchback. Chomosoma 98, 81-85.

Van Vactor, D., Sink, H., Fambrough, D., Tsoo, R. and Goodman, C. S. (1993). Genes that control neuromuscular specificity in Drosophila. Cell 73, 1137-1153.

Wieschaus, E., Nüsslein-Volhard, C. and Kluding, H. (1984). Krüppel, a gene whose activity is required early in the zygotic genome for normal embryonic segmentation. Dev. Biol. 104, 172-186.

Williams, J. A., Bell, J. B. and Carroll, S. B. (1991). Control of Drosophila wing and haltere development by the nuclear vestigial gene product. Genes Dev. 5, 2481-2495.

(Accepted 4 July 1997) 